\title{
On a Wind-Driven, Double-Gyre, Quasi-Geostrophic Ocean Model: Numerical Simulations and Structural Analysis ${ }^{1}$
}

\author{
Jie Shen, ${ }^{*}$ T. Tachim Medjo, $\nmid$ and S. Wang $\ddagger$ \\ * Department of Mathematics, Penn State University, University Park, Pennsylvania 16802; †Department of \\ Mathematics, Florida International University, DM 413 B, University Park, Miami, Florida 33199; and \\ $\ddagger$ Department of Mathematics, Indiana University, Bloomington, Indiana 47405
}

Received July 20, 1998; revised July 13, 1999

\begin{abstract}
The main objectives of this paper are to adapt an efficient and accurate spectralprojection method for a wind-driven, double-gyre, mid-latitude, quasi-geostrophic ocean model, and to study the double-gyre phenomenon from numerical and structural analysis points of view. A number of numerical simulations are carried out and their structural stability and structural transition/bifurcation are investigated using a new dynamical systems theory of two-dimensional incompressible flows. (c) 1999 Academic Press

Key Words: double-gyre; quasi-geostrophic; wind-driven ocean circulation; spectral-projection method; structural stability, structural bifurcation.
\end{abstract}

\section{INTRODUCTION}

We study in this paper the double-gyre phenomenon of large-scale ocean circulation, a typical phenomenon in the northern mid-latitude ocean basins. The double-gyre phenomenon here refers to the two gyre motions (circulations) observed in the ocean basins: one is the sub-polar gyre, and the other is the sub-tropical gyre. These gyres have a typical horizontal scale of about one thousand kilometers. Several main features of the double-gyre ocean circulation have been identified by analyzing the observational data as well as by numerical simulations; see, for instance, Speich and Ghil [30], Jiang et al. [12], and Speich et al. [29]. First, these gyres are dominant and persistent; second, they represent typical

\footnotetext{
${ }^{1}$ The authors are grateful to the associate editor and the referees for their critical comments which have helped to improve this paper. The authors are also grateful to R. Temam for stimulating discussions and encouragement. This work was supported in part for J.S. by NSF-DMS9623020, for T.T.M. by NSF-DMS-9400615 and by the Research Fund of Indiana University, and for S.W. by NAVY-N00014-96-1-0425 and NSF-DMS-9623071. Part of the computations were carried out using facilities provided by the NSF under Grant CDA-9601632.
} 
seasonal and inter-annual oscillations of the large-scale ocean; third, they transfer potential energy. Thus, the study of the double-gyre motion will provide a better understanding of the predictability and, possibly, a better long-term prediction on the dynamics of the ocean.

The main objectives of this paper are:

- to introduce an efficient spectral-projection scheme for the model to simulate typical double-gyre phenomena;

- to analyze the structure and the structural bifurcation of the model using a newly developed dynamical systems theory for incompressible vector fields; and

- to partially examine the effects of the nonlinearity and wind stress to the double-gyre type of circulations.

This study is part of a long-term project, the objectives of which are to document, through careful theoretical studies, the presence of regular inter-annual and inter-decade variability in large-scale ocean basins, to verify the robustness of this variability's characteristics with respect to changes in model parameters, and to investigate the underlying physical mechanisms of the variability. It has also been observed that flow transport and mixing crossing the central jet and anti-cyclonic anomalies as well as symmetry breaking appear frequently; they are phenomena with timescale in years and appear to be typically related to the inter-annual variability associated with the double gyres. One possible explanation for the appearance of anti-cyclonic anomalies and symmetry breaking is the Coriolis force and the nonlinear nature of the problem. A thorough understanding of this variability is essential in determining the climate system's predictability on sub-continental and smaller spatial scales for timescales that equal and exceed a few years.

Although only a simple quasi-geostrophic model is investigated using our numerical method and the new dynamical systems theory, it is hoped that the methods and ideas presented here will be useful in more realistic models as well as in other relevant problems.

We briefly describe our approach below:

First, the model we adapt in the study is a two-dimensional wind-driven, double-gyre, mid-latitude, beta-plane quasi-geostrophic (QG) ocean circulation model. Many published results are available on wind-driven ocean circulation; see, among others, Veronis [33], Pedlosky [22], Stommel [31], Charney [5], Haidvogel et al. [9], Speich and Ghil [30], Jiang et al. [12], Speich et al. [29], Chassignet [6, 7, 21], Holland [10], Holland and Rhines [11], Berloff and Meacham [1, 2], and Meacham and Berloff [20]. They used this model to study the formation of the western boundary currents in the North Pacific and the North Atlantic. Bryan [3] investigated the nonlinear model of the wind-driven ocean and found that there were two regimes depending on the Reynolds numbers. His results suggest that below a critical value between 50 and 100, the flow always converges to a steady-state solution. Above that critical value, instabilities would occur. As in the references mentioned above, the spatial domain we use is $1000 \mathrm{~km}$ (east-west) by $2000 \mathrm{~km}$ (north-south), which is an idealized geometry applicable to the Pacific and Atlantic; similar idealized geometries were also used in all the references mentioned above.

Second, we present and implement an efficient and accurate numerical scheme for the wind-driven, double-gyre, quasi-geostrophic model. More specifically, we discretize the spatial variables by using a spectral-Galerkin method $[25,27]$ which is fast and significantly more accurate than the traditional finite difference or finite element methods, and we advance in time by using a second-order projection scheme (cf. $[8,32,13,27])$ which treats the incompressibility constraint efficiently. 
Third, we apply a new dynamical systems theory for two-dimensional incompressible flows developed recently by Ma and Wang [16, 17] (see also [18]) to analyze the structural stability and structural transitions/bifurcations of the flow patterns in the QG type of model. The point of view of this theory is to classify the topological structure and its transitions of the instantaneous velocity field (i.e., streamlines in the Eulerian coordinates), treating the time variable as a parameter. One justification of this viewpoint is that given initial data, for a residual set (i.e., the intersection of a countable number of open and dense sets) of forcing, the instantaneous velocity field is structurally stable in an open and dense set of the time interval. Hence, the structural bifurcation can occur only on a nowhere dense set of time, and is a well-defined concept. In particular, we will investigate the following topics: (i) the structural stability of the flow patterns in various flow regions, (ii) structural bifurcation of the flow patterns using either the Reynolds number or time as a parameter, and (iii) quasi-periodicity which appears in the more physically relevant case, demonstrating the presence of regular inter-annual variability in the ocean basins.

The paper is organized as follows. We present the model equations in the next section, followed by a spectral-projection scheme for this model in Section 3. Then, we present in Section 4 numerical simulations and study their structural stabilities for a range of physically relevant parameters. Some concluding remarks are given in Section 5. For the readers' convenience, a geometrical theory of two-dimensional incompressible flows is recapitulated in the Appendix.

\section{THE WIND-DRIVEN, DOUBLE-GYRE, QUASI-GEOSTROPHIC MODEL OF LARGE-SCALE OCEAN CIRCULATION}

The model we adapt in our studies is the following $\beta$-plane, wind-driven, double-gyre, quasi-geostrophic model of large-scale ocean circulation,

$$
\left\{\begin{array}{l}
\frac{\partial v}{\partial t}-\frac{1}{\operatorname{Re}} \Delta v+v \cdot \nabla v+\frac{1}{\varepsilon}[y k \times v+\nabla p]=\frac{\alpha_{r}}{\varepsilon \pi} \tau, \\
\operatorname{div} v=0
\end{array}\right.
$$

where $v=(u, w)$ is the (horizontal) velocity field, $p$ is the surface pressure, and $k$ is the unit vector in the vertical direction so $k \times v=(-w, u)$. The spatial coordinate system is denoted by $(x, y)$ with the $x$-axis in the east-west direction and the $y$-axis in the north-south direction. The two-dimensional QG model can be derived from a three-dimensional one by integrating in the vertical direction; we refer the readers to [14] for the mathematical analysis of the issues related to the QG model.

We proceed with the notation used in (2.1).

- The corresponding dimensional form of (2.1) reads

$$
\left\{\begin{array}{l}
\frac{\partial v}{\partial t}-A \Delta v+v \cdot \nabla v+\beta y k \times v+\nabla p=\alpha_{r} \frac{\tau_{\text {dim }}}{\rho_{0} h} \\
\operatorname{div} v=0
\end{array}\right.
$$

Here $\beta y$ represents the first-order Coriolis parameter, and $A$ the effective turbulent viscosity coefficient. The nondimensional parameter $\alpha_{r}$ is introduced here to study the effects of the wind stress on the dynamics; its value can be allowed to range between 0 and 1.8 (equivalent to taking the wind stress from abnormally low to abnormally high, as in [12]). The term 
$\tau_{\mathrm{dim}} / \rho_{0} h$ is dictated by the Ekman pumping on the surface Ekman boundary layer, and is also related to the leading order Coriolis term $f_{0} k \times v$ in the quasi-geostrophic asymptotics; see Pedlosky [23].

- The flow is driven by the wind force, which is given in the dimensional form as a sinusoidal function

$$
\tau_{\operatorname{dim}}=\tau_{0}(-\cos (\pi y / L), 0),
$$

where $\tau_{0}$ is the amplitude of the wind force. The nondimensional form of the wind force used in $(2.1)$ is $\tau=(-\cos (\pi y), 0)$.

- The typical (horizontal) velocity $U$ is calculated via the Sverdrup equation as

$$
U=\frac{\tau_{0} \pi}{\beta L \rho_{0} H},
$$

where $L$ is the typical horizontal length, $H$ is the typical depth of the ocean layer, and $\rho_{0}$ is the typical surface density.

- The parameters $\varepsilon$ and Re are the Rossby number and the Reynolds number given by

$$
\varepsilon=\frac{U}{\beta L^{2}}, \quad \operatorname{Re}=\frac{L U}{A},
$$

where $A$ is the effective turbulent viscosity coefficient. The values of the parameters used in this work are

$$
\begin{array}{rlrl}
L & =10^{6} \mathrm{~m}, & H=500 \mathrm{~m}, \\
f_{0}=5 \times 10^{-5} \mathrm{~s}^{-1}, & & \beta=2 \times 10^{-11} \mathrm{~m}^{-1} \cdot \mathrm{s}^{-1}, \\
\tau_{0} & =0.1 \mathrm{~N} \cdot \mathrm{m}^{-2}, & & \rho_{0}=1000 \mathrm{~kg} \cdot \mathrm{m}^{-3}, \\
U & =10^{-2} \pi \mathrm{m} \cdot \mathrm{s}^{-1}, & & \varepsilon=5 \times 10^{-4} \pi .
\end{array}
$$

- The dimensional time and velocity are calculated by

$$
t_{\mathrm{dim}}=\frac{1}{\varepsilon \beta L} t=\frac{10^{8}}{\pi} t \mathrm{~s}, \quad v_{\mathrm{dim}}=U v .
$$

We take the nondimensional spatial domain $M$ and the nondimensional depth $h$ of the ocean to be

$$
M=(0,1) \times(0,2) \subset \mathbb{R}^{2}, \quad h=1 .
$$

The nondimensional form of the boundary conditions and the initial conditions is

$$
\left\{\begin{array}{ll}
v \cdot n=0, & \frac{\partial(v \cdot \tau)}{\partial n}=0 \\
\left.v\right|_{t=0}=0 & \text { on } M .
\end{array} \text { on } \partial M,\right.
$$

\section{A SPECTRAL-PROJECTION METHOD}

\subsection{Time Discretization-A Second-Order Projection Scheme}

One of the main difficulties in the numerical simulation of Eqs. (2.1)-(2.4), or more generally, equations describing any incompressible viscous flows, is associated with the 
incompressibility constraint. The projection method, initially proposed by Chorin [8] and Temam [32], was designed to overcome this difficulty. We propose to use the following scheme which is a second-order variant (cf. [27]) of the original projection method,

$$
\left\{\begin{array}{l}
\frac{3 \hat{v}^{m+1}-4 v^{m}+v^{m-1}}{2 \Delta t}-\frac{1}{\operatorname{Re}} \Delta \hat{v}^{m+1}+f^{m+1} k \times \hat{v}^{m+1}=F^{m+1}-\nabla p^{m}-\left\{2 N L T^{m}-N L T^{m-1}\right\}, \\
\hat{v}^{m+1} \cdot n=0, \quad \frac{\partial\left(\hat{v}^{m+1} \cdot \tau\right)}{\partial n}=0 \quad \text { on } \partial M
\end{array}\right.
$$

and

$$
\left\{\begin{array}{l}
\frac{3\left(v^{m+1}-\hat{v}^{m+1}\right)}{2 \Delta t}+\nabla\left(p^{m+1}-p^{m}\right)=0, \\
\operatorname{div} v^{m+1}=0, \\
v^{m+1} \cdot n=0 \quad \text { on } \partial M,
\end{array}\right.
$$

where $N L T=(v \cdot \nabla) v$, and for the sake of generality, we have replaced $(1 / \varepsilon) y$ and $\left(\alpha_{\tau} / \varepsilon \pi\right) \tau$, respectively, by generic functions $f$ and $F$.

Taking the divergence of the first equation in (3.2), we find that (3.2) is equivalent to

$$
\left\{\begin{array}{l}
-\Delta\left(p^{m+1}-p^{m}\right)=-\frac{3}{2 \Delta t} \operatorname{div} \hat{v}^{m+1} \\
\frac{\partial\left(p^{m+1}-p^{m}\right)}{\partial n}=0 \quad \text { on } \partial M \\
v^{m+1}=\hat{v}^{m+1}-\frac{2 \Delta t}{3} \nabla\left(p^{m+1}-p^{m}\right) .
\end{array}\right.
$$

We can write the above equations in components form as follows: Setting $v=(u, w), \hat{v}=$ $(\hat{u}, \hat{w}), N L T=\left(N L T_{1}, N L T_{2}\right), F=\left(F_{u}, F_{w}\right)$, and

$$
\alpha=\frac{3 \operatorname{Re}}{8 \Delta t}, \quad \beta=\frac{1}{4}, \quad \gamma=\frac{\operatorname{Re}}{4}, \quad \sigma=\frac{3}{8 \Delta t}, \quad \alpha_{1}=\frac{\operatorname{Re}}{8 \Delta t},
$$

multiplying (3.1) by $\gamma$ and (3.2) by $\frac{1}{4}$, then, after mapping the domain $M=(0,1) \times(0,2)$ to the computational domain $D=(-1,1) \times(-1,1)$, we obtain

$$
\begin{aligned}
& \begin{cases}\alpha \hat{u}^{m+1}-\hat{u}_{x x}^{m+1}-\beta \hat{u}_{y y}^{m+1}-\gamma f^{m+1} \hat{w}^{m+1} & \\
=\alpha_{1}\left(4 u^{m}-u^{m-1}\right)+\gamma\left\{F_{u}^{m+1}-2 p_{x}^{m}-\left(2 N L T_{1}^{m}-N L T_{1}^{m-1}\right)\right\}, & \text { in } D, \\
\hat{u}^{m+1}=0 \quad \text { at } x= \pm 1, \quad\left(\hat{u}^{m+1}\right)_{y}=0 \quad \text { at } y \pm 1 ;\end{cases} \\
& \begin{cases}\alpha \hat{w}^{m+1}-\hat{w}_{x x}^{m+1}-\beta \hat{w}_{y y}^{m+1}+\gamma f^{m+1} \hat{u}^{m+1} & \text { in } D, \\
=\alpha_{1}\left(4 w^{m}-w^{m-1}\right)+\gamma\left\{F_{w}^{m+1}-p_{y}^{m}-\left(2 N L T_{2}^{m}-N L T_{2}^{m-1}\right)\right\}, & \text { at } y \pm 1 ; \\
\hat{w}_{x}^{m+1}=0 \quad \text { at } x= \pm 1, \quad \hat{w}^{m+1}=0 \quad \text { in } D\end{cases} \\
& \begin{cases}-\left(p^{m+1}-p^{m}\right)_{x x}-\beta\left(p^{m+1}-p^{m}\right)_{y y}=-\sigma\left(2 \hat{u}_{x}^{m+1}+\hat{w}_{y}^{m+1}\right), & \text { on } \partial D \\
\frac{\partial\left(p^{m+1}-p^{m}\right)}{\partial n}=0 \quad & \end{cases}
\end{aligned}
$$

and

$$
\left\{\begin{array}{l}
u^{m+1}=\hat{u}^{m+1}-\frac{4 \Delta t}{3}\left(p^{m+1}-p^{m}\right)_{x} \\
w^{m+1}=\hat{w}^{m+1}-\frac{2 \Delta t}{3}\left(p^{m+1}-p^{m}\right)_{y}
\end{array}\right.
$$


The main advantage of this approach is that we only need to solve a coupled elliptic system (3.4)-(3.5) for the velocity components and a Poisson equation (3.6) at each time step, whereas a direct discretization of (2.1)-(2.4) would require us to solve a coupled, nonpositive-definite system for $(u, w, p)$. Furthermore, the above scheme can be extended to three-dimensional cases in a straightforward manner.

Remark 3.1. The two velocity components $\left(\hat{u}^{m+1}, \hat{w}^{m+1}\right)$ are coupled together by the Coliolis force. Although the coupled system (3.4)-(3.5) is elliptic so it can be solved by using standard direct or iterative procedures, the presence of nonconstant coefficient $f$ prevents us from using existing fast solvers for Poisson-type equations. An alternative is to treat the Coliolis force explicitly in (3.4)-(3.5), leading to two decoupled Poisson-type equations with constant coefficients for $\hat{u}^{m+1}$ and $\hat{w}^{m+1}$ which can then be solved by existing fast solvers. The disadvantage of treating the Coliolis force explicitly is that it may lead to a more rigid time step constraint which may offset the savings realized by using fast solvers at each time step. Hence, whether to treat the Coliolis force explicitly or implicitly depends on many factors related to the choice of spatial discretizations and availablity of fast solvers.

\subsection{Space Discretization-Spectral-Galerkin Method}

Although we can solve Eqs. (3.4), (3.5), and (3.6) by using a finite difference method (see, for instance, [28]), to fully take advantage of the simple geometry in hand, we propose to use a spectral method which is capable of providing much more accurate results using a relatively smaller number of unknowns. Since the spectral-Galerkin method developed in $[25,26]$ is extremely efficient for elliptic equations with constant coefficients, we chose to treat the Coliolis force explicitly in (3.4)-(3.5) so that we can use the fast spectralGalerkin method. To fix the idea, let us present in some detail the Legendre-Galerkin for the equation

$$
\left\{\begin{array}{l}
\alpha u-u_{x x}-\beta u_{y y}=f, \quad \text { in } D, \\
\left.u\right|_{x= \pm 1}=0,\left.\quad u_{y}\right|_{y= \pm 1}=0,
\end{array}\right.
$$

which corresponds to (3.4) with $\hat{w}^{m+1}$ treated explicitly. The other two equations can be solved similarly.

Let $P_{K}$ be the space of all polynomials of degree less than or equal to $K$. We set

$$
\begin{aligned}
X_{N} & =\left\{w \in P_{N}: w( \pm 1)=0\right\} \\
Y_{M} & =\left\{w \in P_{M}: w_{y}( \pm 1)=0\right\} \\
Z_{N M} & =X_{N} \times Y_{M} .
\end{aligned}
$$

Then, our spectral-Galerkin method is to look for $u_{N M} \in Z_{N M}$ such that

$$
\alpha\left(u_{N M}, v\right)+\left(\partial_{x} u_{N M}, \partial_{x} v\right)+\beta\left(\partial_{y} u_{N M}, \partial_{y} v\right)=(f, v), \quad \forall v \in Z_{N M},
$$

where $(u, v)=\int_{D} u v d x d y$.

The efficiency of the method depends on the choice of basis function for $X_{N}$ and $Y_{M}$. It is easy to verify that for 


$$
\begin{aligned}
& \phi_{i}(x)=\frac{1}{\sqrt{4 i+6}}\left(L_{i}(x)-L_{i+2}(x)\right), \\
& \rho_{j}(y)=\sqrt{\frac{(j+2)(j+3)}{j(j+1)(4 j+6)}}\left(L_{j}(y)-\frac{j(j+1)}{(j+2)(j+3)} L_{j+2}(y)\right),
\end{aligned}
$$

where $L_{l}(s)$ is the $l$ th-degree Legendre polynomial, we have

$$
X_{N}=\operatorname{span}\left\{\phi_{i}(x): i=0,1, \ldots, N-2\right\}, \quad Y_{M}=\operatorname{span}\left\{\rho_{j}(y): j=0,1, \ldots, M-2\right\},
$$

and

$$
\int_{-1}^{1} \phi_{i}^{\prime}(x) \phi_{j}^{\prime}(x) d x=\delta_{i j}, \quad \int_{-1}^{1} \rho_{i}^{\prime}(y) \rho_{j}^{\prime}(y) d y=\delta_{i j} .
$$

Hence, setting

$$
\begin{aligned}
u_{N M} & =\sum_{i=0}^{N-2} \sum_{j=0}^{M-2} u_{i j} \phi_{i}(x) \rho_{j}(y), \\
a_{i j} & =\int_{-1}^{1} \phi_{j}(x) \phi_{i}(x) d x, \\
b_{i j} & =\int_{-1}^{1} \rho_{j}(y) \rho_{i}(y) d y, \quad f_{i j}=\int_{D} f \phi_{i}(x) \rho_{j}(y) d x d y,
\end{aligned}
$$

and letting $A, B, F$, and $U$ be the corresponding matrices with entries given above, then (3.10) is equivalent to the following matrix system:

$$
\alpha A U B+U B+\beta A U=F
$$

Note that the matrices $A$ and $B$ are symmetric and have only three nonzero diagonals. Hence, (3.12) can be efficiently solved by using the matrix decomposition method [15, 27] at a cost of $4 N M \min (N, M)+O(N M)$ operations.

Remark 3.2. It is also possible to solve (3.8) by using the Chebyshev-Galerkin method [26], which allows the use of the fast Fourier transform, or the Chebyshev-LegendreGalerkin method [27], which takes advantage of both the Chebyshev and the Legendre polynomials. Thus, the spectral-Galerkin method is capable of solving Eqs. (3.4)-(3.6) at a cost comparable to the traditional finite difference method with the same number of unknowns. Thus, thanks to the superior accuracy of the spectral discretization [4], the use of the spectral-Galerkin method would reduce the degree of freedom needed for a particular problem and hence result in a significant savings in CPU time.

\section{NUMERICAL RESULTS AND STRUCTURAL ANALYSIS OF THE DOUBLE-GYRE OCEAN CIRCULATION}

\subsection{Numerical Results}

In our numerical experiments, we use $\alpha_{r}=0.95$ with Reynolds numbers $5,10,20$, and 30 , respectively. 

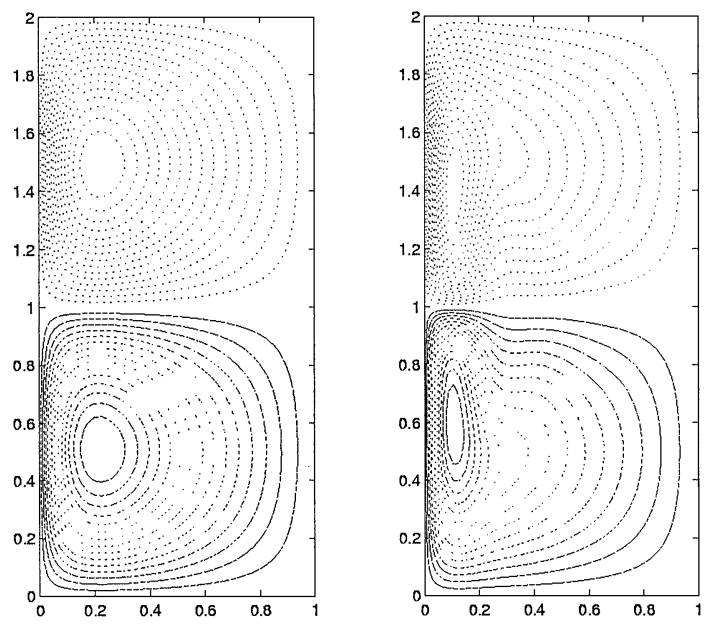

FIG. 4.1. Snapshots of streamlines for $\operatorname{Re}=5$ at time $t=0.01$ year and at the steady state.

All computations are carried out using a grid size of $49 \times 65$, and a (nondimensional) time step $\Delta t=10^{-4}$, which corresponds to the dimensional time step $\Delta t_{\mathrm{dim}}=10,000 / \pi \mathrm{s} \approx$ $53.05 \mathrm{~min}$. Note that the maximum allowable (nondimensional) time step is about $10^{-3}$ for $\mathrm{Re}=5$ to $1.5 \times 10^{-4}$ for $\mathrm{Re}=30$.

Figures 4.1-4.3 show respectively the snapshots of streamliness at $\operatorname{Re}=5,10$, and 20 . As evidenced by Fig. 4.4, the flows at these Reynolds numbers converge to steady-state solutions. Figures 4.1-4.3 indicate that the steady states are characterized by two gyres; one is cyclonic in the northern part of the basin and another is anti-cyclonic in the southern part. The two gyres are separated by a meandering jet; see also [3].

For $\mathrm{Re}=30$, the flow never settled down to a steady state as indicated by Fig. 4.5. The phase portrait and spectral density of the kinetic energy history in Fig. 4.6 indicate that the flow becomes essentially quasi-periodic with a few incommensurate frequencies. Figures 4.7-4.10 are snapshots of the streamlines at various times.
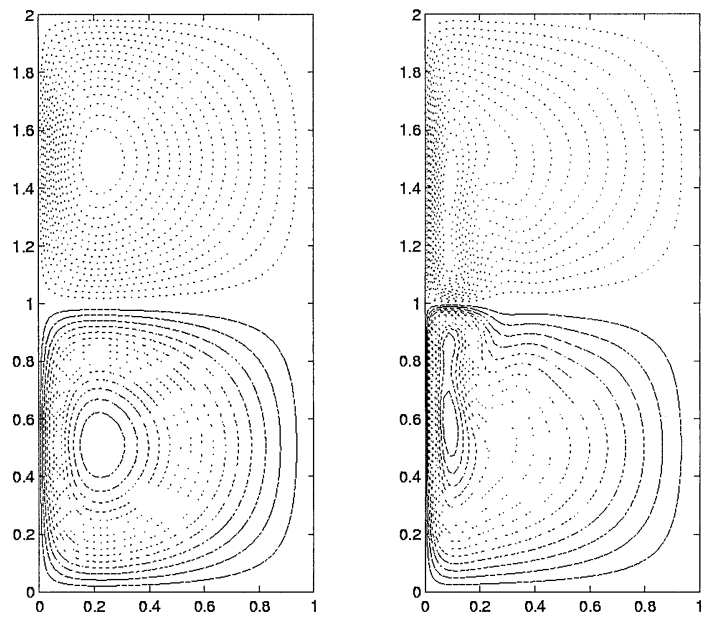

FIG. 4.2. Snapshots of streamlines for $\operatorname{Re}=10$ at time $t=0.01$ year and at the steady state. 

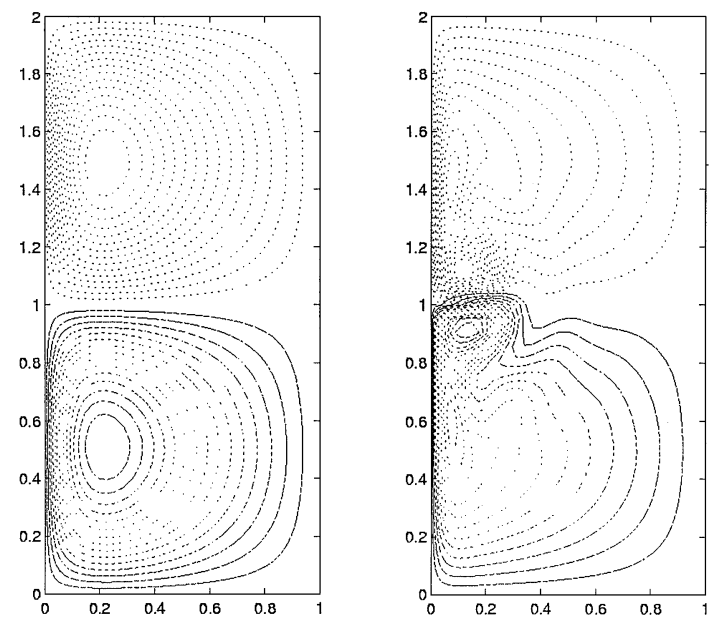

FIG. 4.3. Snapshots of streamlines for $\operatorname{Re}=20$ at time $t=0.01$ year and at the steady state.
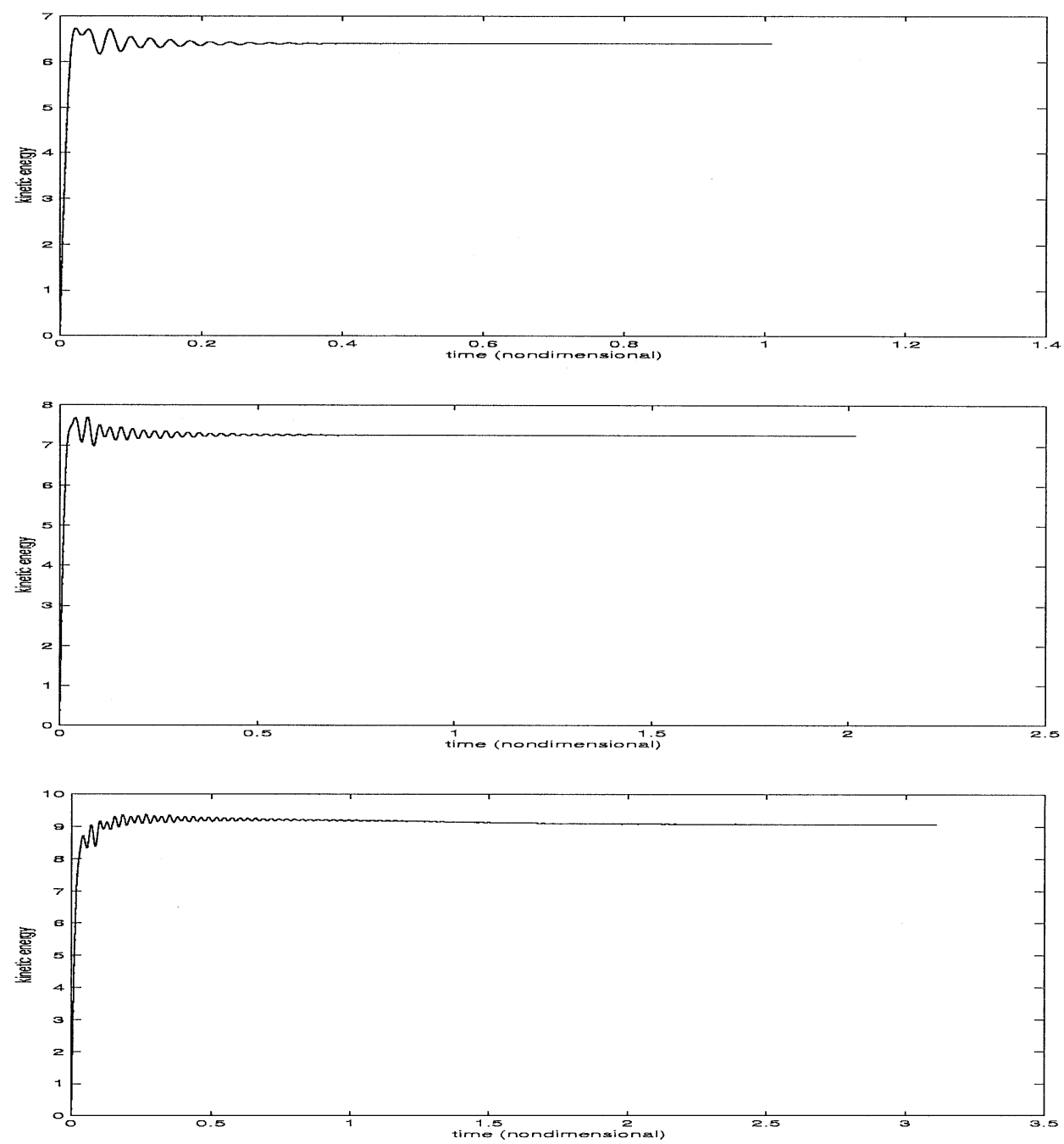

FIG. 4.4. Convergence histories of kinetic energy for $\operatorname{Re}=5,10,20$. 

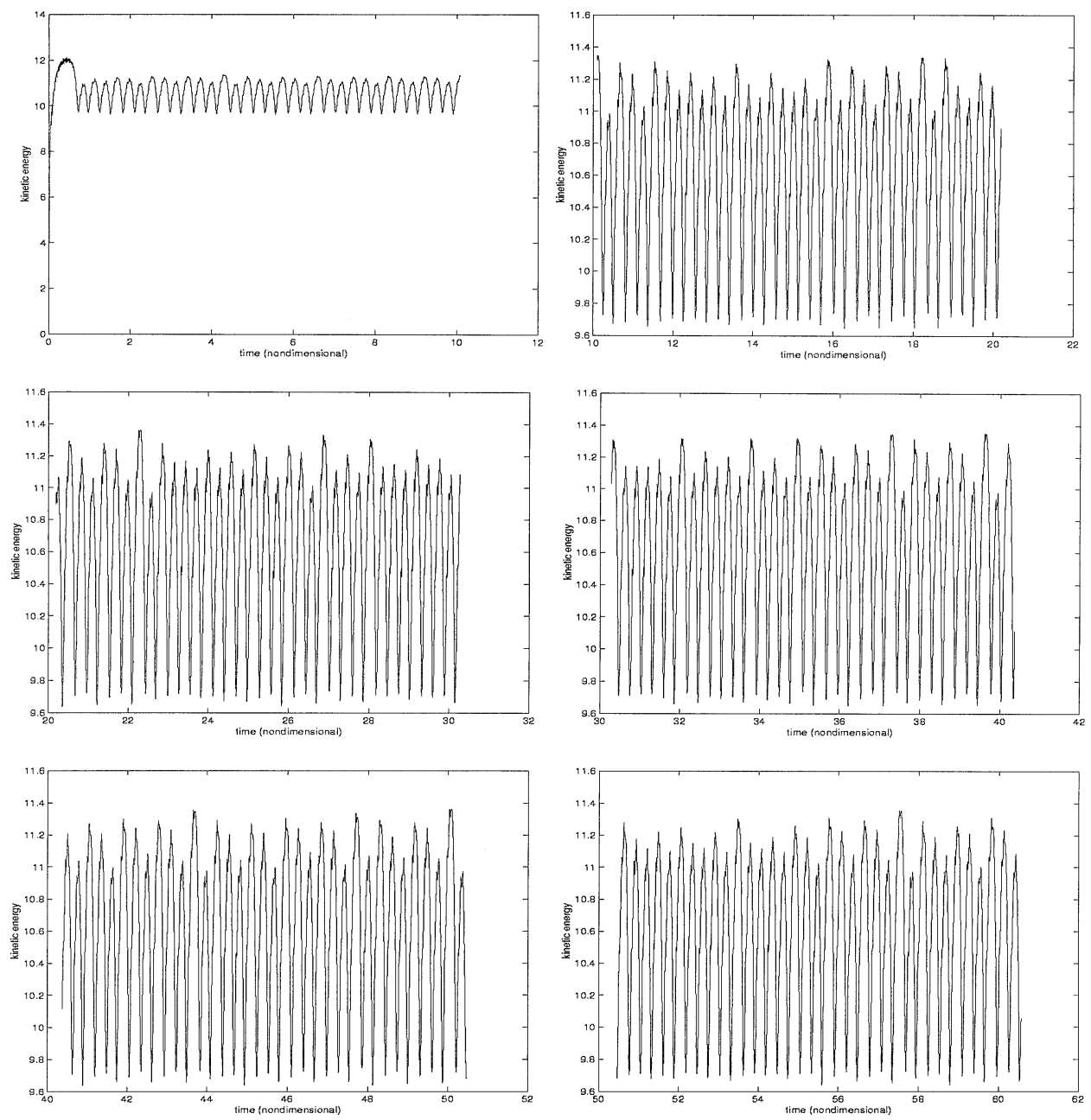

FIG. 4.5. Convergence history of kinetic energy, $\mathrm{Re}=30$.
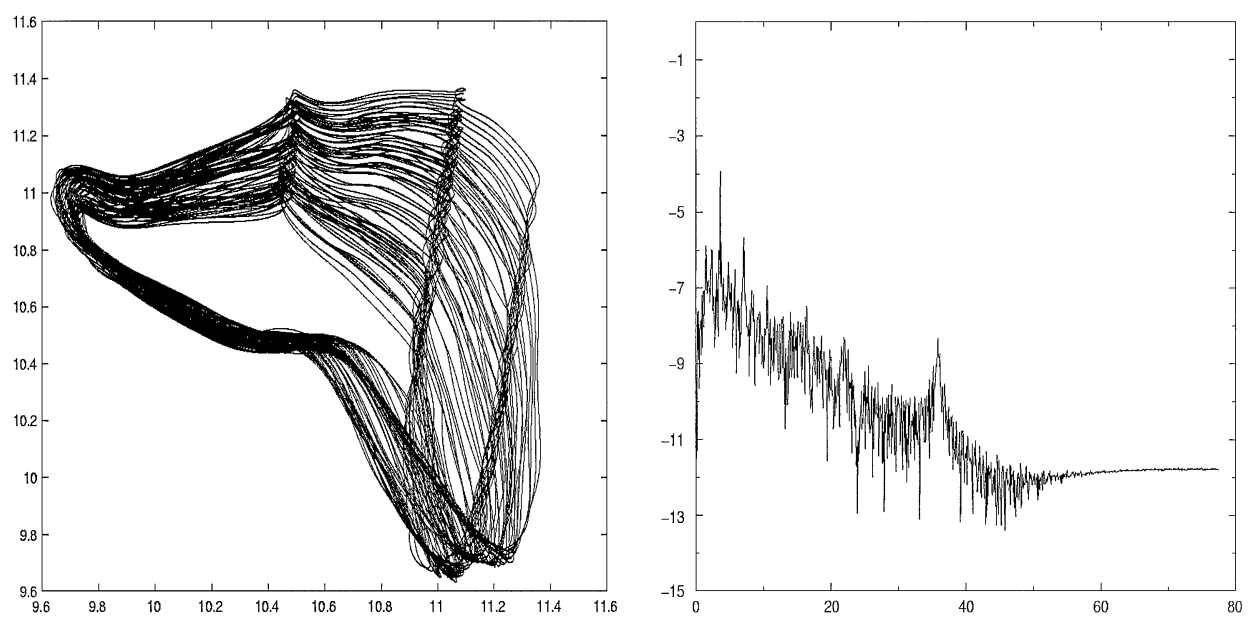

FIG. 4.6. Phase portrait and spectral density of the kinetic energy at $\operatorname{Re}=30$. 

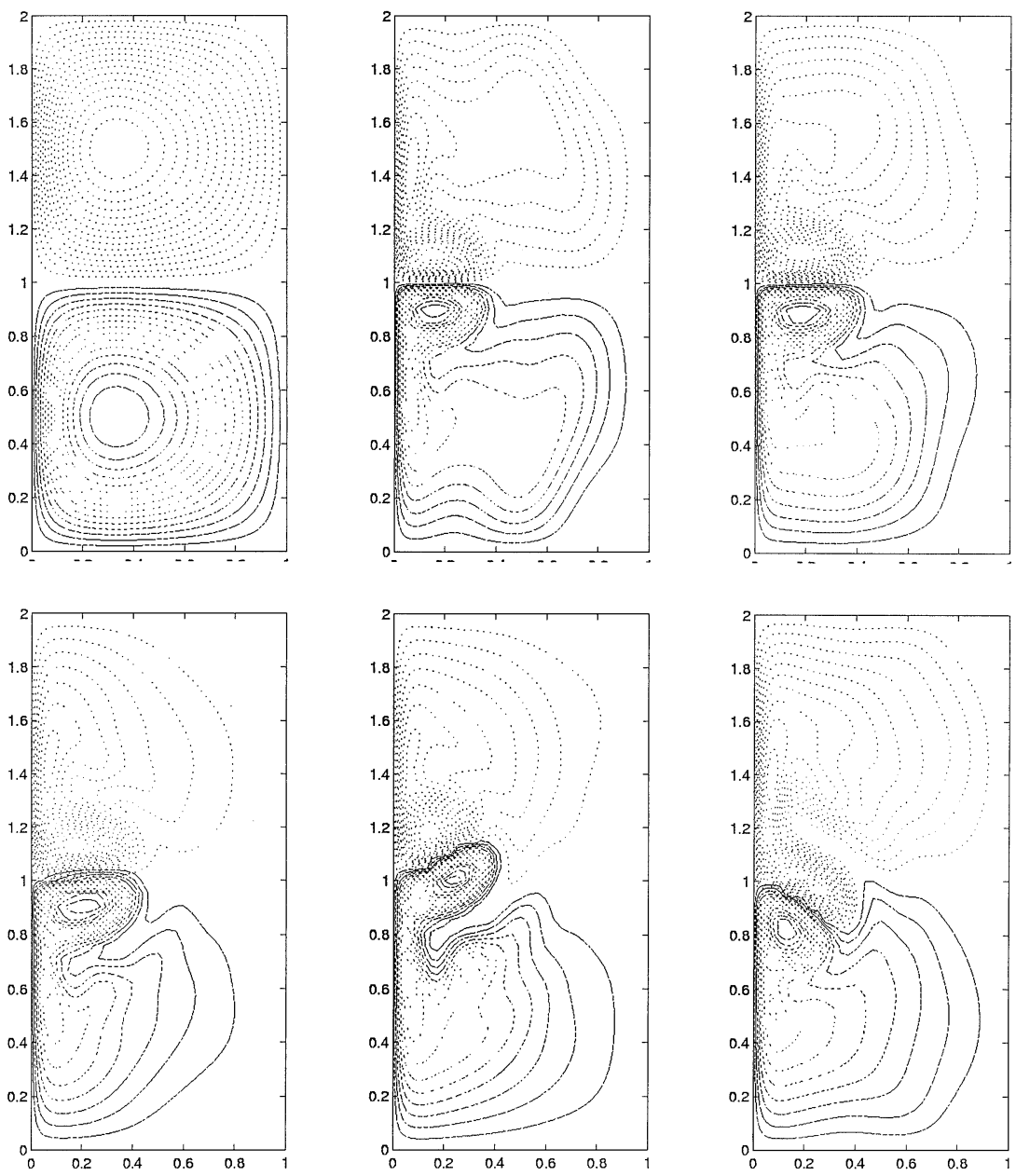

FIG. 4.7. Snapshots of streamlines for $\operatorname{Re}=30$, at time $t=0.005, t=0.11, t=0.20, t=0.44, t=0.63$, and $t=0.87$ year.

\subsection{Structure Analysis of the Double-Gyre Ocean Circulation}

One of the main objectives of this paper is to apply the newly developed dynamical systems theory for incompressible flows to analyze the structure and bifurcation of the double-gyre phenomenon of the mid-latitude ocean basins. The point of view of this theory is to classify the topological structure and its transitions of the instantaneous velocity field (i.e., streamlines in the Eulerian coordinates), treating the time variable as a parameter. One justification of this viewpoint is that given initial data, for a residual set (i.e., the intersection of a countable number of open and dense sets) of forcing, the instantaneous velocity field is structurally stable in an open and dense set of the time interval. Hence, the structural bifurcation can occur only on a nowhere dense set of time, and structural bifurcation is therefore a well-defined concept. We summarize this theory below and refer to the Appendix or [16-18] for more details.

- Classification of singularities: Thanks to the divergence-free condition of the velocity field, a nondegenerate interior singular point must be either a saddle or a center, and a boundary singular point must be a saddle. In the oceanic setting, a center corresponds to 

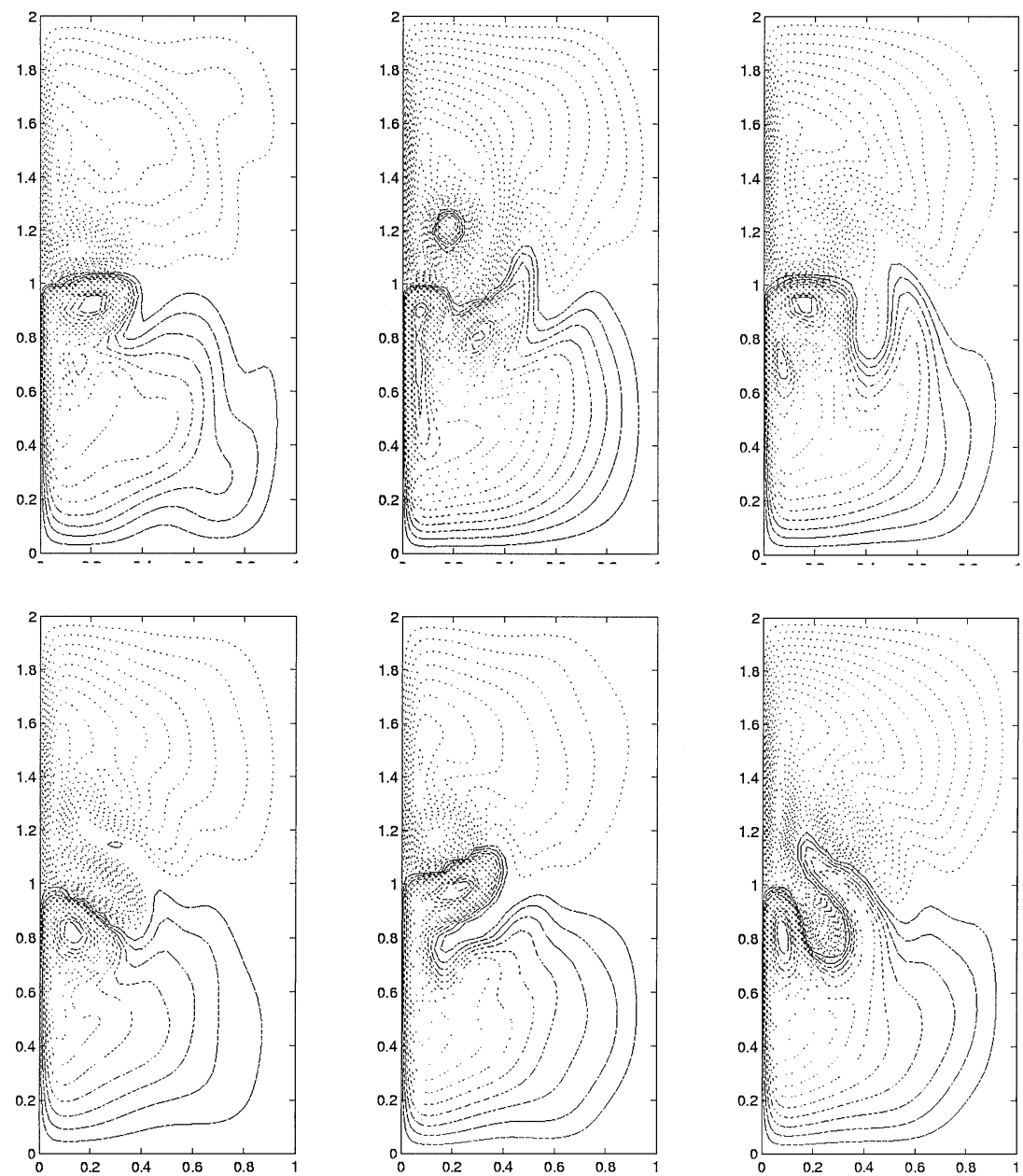

FIG. 4.8. Snapshots of streamlines for $\operatorname{Re}=30$, at time $t=1.04, t=1.23, t=1.52, t=2.02, t=2.26$, and $t=2.64$ years.

a circulation center of the flow; a saddle corresponds to the intersection of two jets moving toward each other; and a boundary saddle is due to a jet moving toward or leaving the boundary. The index formula (A.4) in the Appendix links the number of centers $C$, the number of interior saddles $S$, and the number of boundary saddles $B$ with the number of islands $k$ in the basin. In particular, for the double-gyre problem we address in this paper we have $k=0$, and (4.1) below holds true. As we can see from the numerical results presented, centers and boundary saddles are observable. Therefore, the number of interior saddles is determined easily by (A.4), so is the phase structure.

- Structural classification: The topological set of orbits of a regular divergence-free velocity field consists of a finite number of connected components of circle cells, circle bands, and saddle connections. Each circle cell is the largest circulation region around a center. The boundary of a circle cell consists of saddle connections. A circle band is a band of invariant flow region, where the flow lines are periodic and the flow meanders in the band.

- Topological classification: The global topological structure of the flow orbits of a regular divergence-free vector field is completely determined by its saddle connection set. 

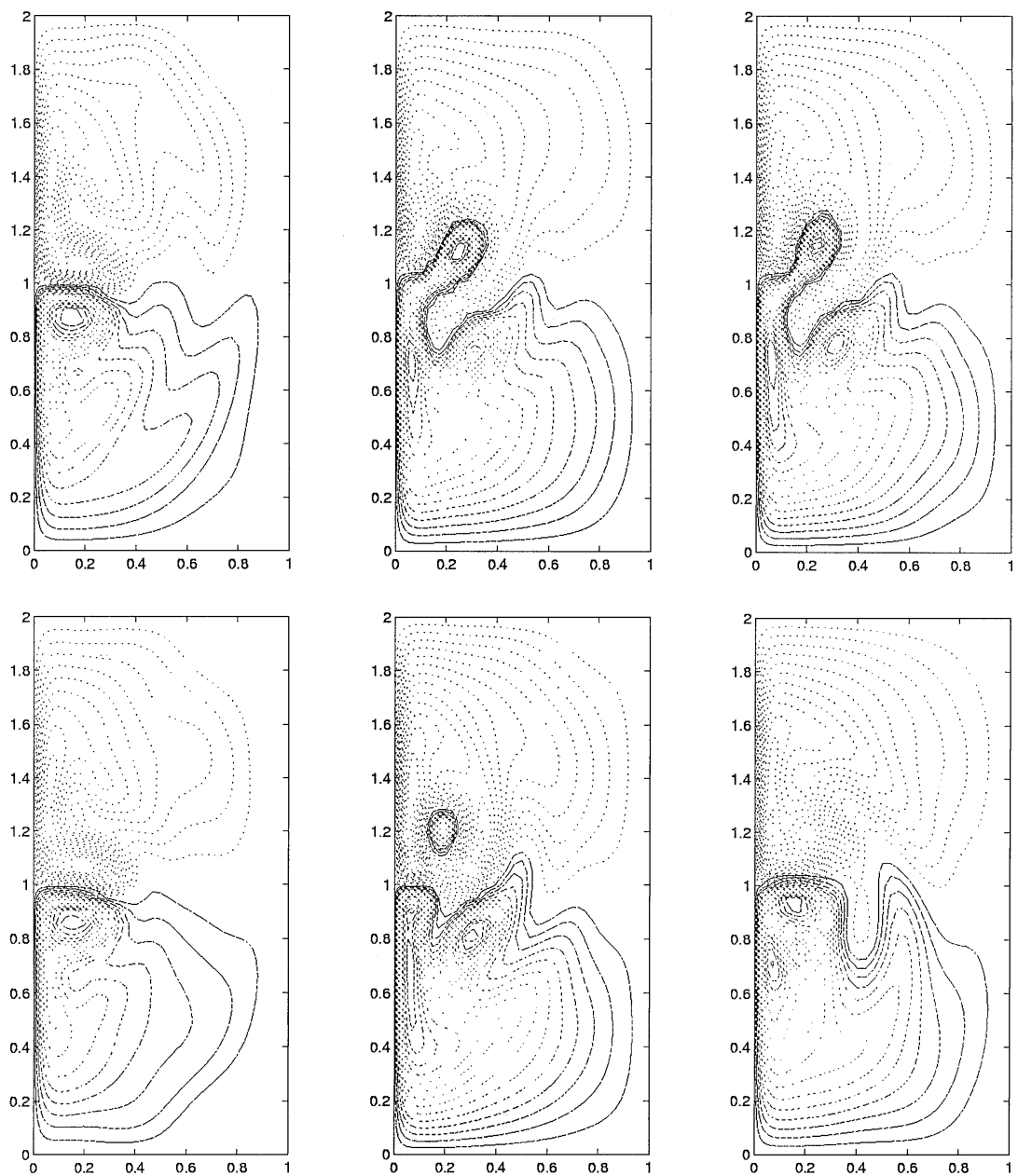

FIG. 4.9. Snapshots of streamlines for $\operatorname{Re}=30$ at time $t=3.03, t=3.51, t=4.08, t=4.81, t=5.85$, and $t=7.30$ years.

- Structural stability: Notice that the divergence-free condition changes completely the general features of structurally stable fields as compared to the situation when this condition is not present. The latter case was studied in a classical paper of Peixoto [24]. The conditions for structural stability and genericity in Peixoto's theorem are: (i) the field can have only a finite number of singularities and closed orbits (critical elements) which must be hyperbolic; (ii) there are no saddle connections; and (iii) the nonwandering set consists of singular points and closed orbits.

The structural stability theorem developed by Ma and Wang states that a divergence-free vector field $v$ is structurally stable with respect to divergence-free vector field perturbations if and only if (1) $v$ is regular, (2) all interior saddle points of $v$ are self-connected, and (3) each boundary saddle point is connected to boundary saddles on the same connected component of the boundary.

The first condition here requires only regularity of the field and so it does not exclude centers which are not hyperbolic and excluded by (i) above. The second condition is of a completely different nature from the corresponding one in the Peixoto theorem. Namely, condition (ii) above excludes the possibility of saddle connections. In contrast, (2) amounts 

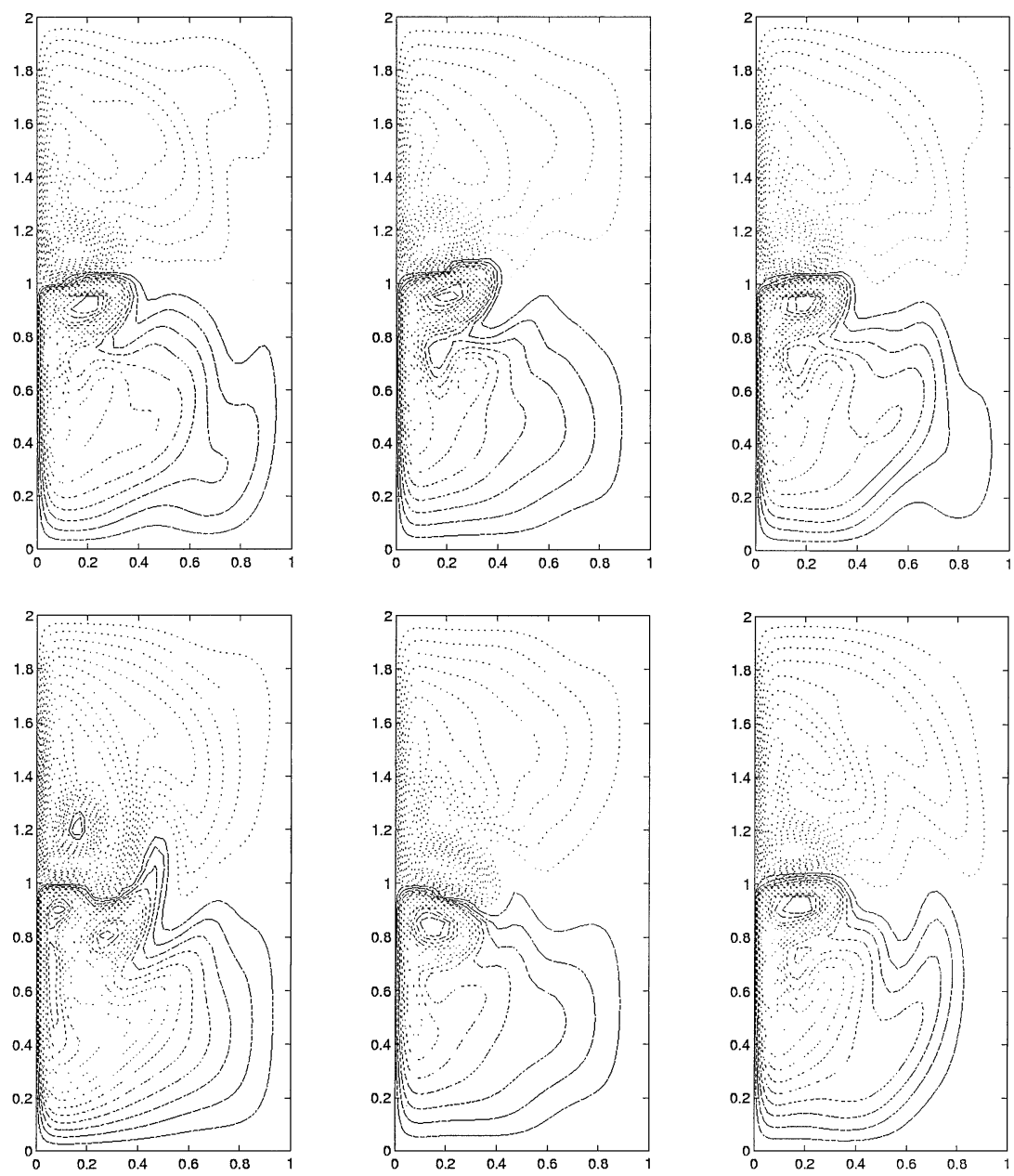

FIG. 4.10. Snapshots of streamlines for $\operatorname{Re}=30$ at time $t=70.4, t=70.56, t=71.04, t=71.23, t=71.41$, and $t=71.60$ years.

to saying that all interior saddles are self-connected! Namely, the interior saddles occur in graphs whose topological form is that of the number 8 , the singularities themselves being hyperbolic. Condition (3) deals with singularities on the boundary.

- Genericity of solutions of fluid equations: Ma and Wang proved in [19] that for an open and dense sub-set of forcing in proper function space, all steady-state solutions of the two-dimensional Navier-Stokes equations as well as (2.1) studied in this paper are structurally stable. Moreover, given initial data, for a residual set (i.e., the intersection of a countable number of open and dense sets) of forcing, the instantaneous velocity field is structurally stable in an open and dense set of the time interval. Hence, the structural bifurcation can occur only on a nowhere dense set of time, and is a well-defined concept.

Hereafter, we shall combine the numerical simulations and the aforementioned dynamical systems theory to analyze the structure and its bifurcation of the double-gyre wind-driven ocean circulation.

General consideration. Consider a mid-latitude ocean basin which occupies a simply connected physical domain as a two-dimensional disk $M \subset \mathbb{R}^{2}$. Let $v \in D^{r}(T M)(r \geq 1)$ be 
regular. In this case, $k$ in (A.4) is zero. Therefore, (A.4) amounts to saying that

$$
C-S-\frac{1}{2} B=1,
$$

where $C, S$, and $B$ are respectively the number of centers, saddle points, and boundary saddle points in the domain.

Consider the large-scale motion of the mid-latitude ocean basins with a strong zonal jet. Because of this jet, there exist two saddle points on the boundary for a nondegenerate surface velocity field: one is on the eastern boundary and the other is on the western boundary. In this case, Formula (4.1) shows that the number of centers equals the number of interior saddle points plus 2. Therefore, the flow with the least singular points contains two centers and no saddle points in the interior. In this case, by the structural stability criterion, Theorem A.3, this flow pattern is structurally stable. In other words, this structure will persist when the time $t$ (or the Reynolds number) changes. The results stated in Section A.7 clearly show that the solution will be structurally stable in an open and dense set of time intervals, and structural bifurcation (with $t$ as a parameter) will occur only at a nowhere dense set of time instants. This justifies the study of the structural analysis of the flow in this paper.

We now analyze below in more detail the structural stability and bifurcation for each of our simulations.

Case $\operatorname{Re}=5$. As we can see in Fig. 4.1, the flow, driven by the wind stress, generates two circular gyres at time $t=0.01$ year. This flow is structurally stable. The flow quickly approaches a steady state of the QG equations. This steady state has the same topological structure as the two circular gyre patterns at $t=0.01$ year, and there is no structural bifurcation present after $t=0.01$ year with respect to the time variable. In view of the structure classification, the phase structure of this pattern consists of two circle cells (two gyres) and the saddle connections.

Case $\operatorname{Re}=10$. The structure of the flow at $\operatorname{Re}=10$ behaves similarly to the flow at $\operatorname{Re}=5$.

Case $\operatorname{Re}=20$. There is a structural bifurcation in the time interval $(0.01,2.03)$. The flow also approaches a steady state as indicated by the energy history in Fig. 4.4. However, the steady state in this case is topologically equivalent to the schematic pattern in Fig. 4.11b. The flow pattern has six centers, four interior saddles, and two boundary saddles, and the index formula holds true in this case. It is easy to see that the flow pattern is structurally stable by the structural stability theorem.

When the Reynolds number is small, the flow converges quickly to a unique steady state, which is structurally stable. Meanwhile, as the Reynolds number changes but remains small, the structural bifurcation would take place. For example, there is one bifurcation value of the Reynolds number between 5 and 20.

Case $\operatorname{Re}=30$. The energy history in Fig. 4.5 shows a quasi-periodic motion; the corresponding dominant frequencies indicate inter-seasonal and inter-annual changes of the double-gyre phenomenon. The energy spectrum and the phase portrait, shown in Fig. 4.6, also suggest that the flow is quasi-periodic, although the dominant frequencies are superimposed with turbulent small-scale motions.

From the point of view of structural analysis, the flow patterns in the first 0.87 year given in Fig. 4.7 indicate several structural transitions/bifurcations between structurally stable 

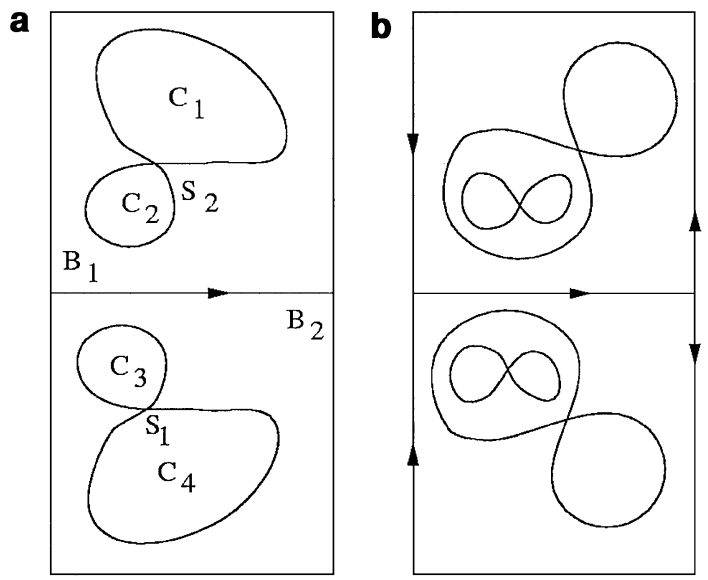

FIG. 4.11. Schematic topological structures.

patterns, and a symmetry breaking of the structure between the sub-polar and sub-tropical cells. Consider now the flow pattern in Figs. 4.8-4.10. The time is sufficiently long to generate typical flow patterns related to long-term dynamics. We can observe from these snapshots the following typical features:

- patterns which are topologically equivalent to those shown in Fig. 4.11 appear frequently, representing the typical gyre circulation;

- several structural transitions/bifurcations between structurally stable patterns appear during this time interval as well as in the beginning period;

- there is a symmetry breaking of the structure between the sub-polar and sub-tropical cells;

- the mixing phenomenon is a typical feature; and

- as the mixing forces sub-gyre circulation detachment, the anti-cyclonic anomalies appear in both the sub-polar and the sub-tropical circulations.

\section{CONCLUDING REMARKS}

To understand the mechanism of the double-gyre phenomenon, a wind-driven, doublegyre, mid-latitude, $f$-plane, quasi-geostrophic ocean model is studied. First, we adapted an efficient and accurate spectral-projection method for this model. The method was based on a spectral-Galerkin approximation (cf. [25] for the spatial variables and a second-order projection scheme (cf. $[13,27]$ ) for time marching. We implemented this method to simulate the wind-driven double-gyre ocean flow for a range of physically relevant parameters.

We used a new dynamical systems theory for two-dimensional incompressible flows $[16,17]$ (see also [18]) to analyze the structural stability and transition of the flow patterns in our numerical simulations. Our analysis led to the following observations:

1. When the Reynolds number is small, the flow converges quickly to a unique steady state, which is structurally stable. Meanwhile, as the Reynolds number changes but remains 
small, the structural bifurcation would take place. For example, there is one bifurcation value of the Reynolds number between 5 and 20 .

2. For $\mathrm{Re}=30$ which is more physically relevant to the double-gyre phenomenon, the flow pattern seems to be quasi-periodic with a few dominant frequencies superimposed with turbulent small-scale motions; the corresponding dominant frequencies indicate interseasonal and inter-annual changes of the double-gyre phenomenon. It is also observed that (i) several structural transitions/bifurcations between structurally stable patterns appear during this time interval as well as in the beginning period; (ii) there is a symmetry breaking of the structure between the sub-polar and sub-tropical cells; (iii) the mixing phenomenon is a typical feature; and (iv) as the mixing forces the detachment of sub-gyre circulation, the anti-cyclonic anomalies appear in both the sub-polar and the sub-tropical circulations.

The anti-cyclonic anomalies and symmetry breaking seem to be generated by the Coriolis force and the nonlinear interaction of the flow.

In summary, our numerical results and their structural analysis demonstrated the presence of regular inter-annual variability in the ocean basins. Anti-cyclonic anomalies appear frequently with timescale in years, and they appear to be typically related to the interannual variability associated with the double gyres. The new dynamical systems theory demonstrates among other things the following features of the problem:

1. Structural bifurcation does occur with Reynolds as a parameter when the steady state is unique.

2. With time $t$ as a parameter and for generic forcing, the time-dependent solution is structurally stable for $t$ in an open and dense set of the entire time interval. This justifies the notion of the structural bifurcation, which appears only for time in a nowhere dense set of the time interval.

3. The flow patterns are structurally classified, providing theoretical possible guidance to both numerical and physical considerations of the problem.

As we mentioned in the Introduction, a simple quasi-geostrophic model is studied in this article with the hope that the methods and ideas presented here will be useful in more realistic models as well as in other relevant problems.

\section{APPENDIX}

\section{Recapitulation of the Geometrical Theory of Two-Dimensional Incompressible Flows}

\section{A.1. General Approach}

Fluid motion and states are normally described by either the Eulerian approach or the Lagrangian approach. For the Eulerian method, one solves the Navier-Stokes type of partial differential equations governing the motion and states of the fluid. The Lagrangian representation of a fluid flow, on the other hand, amounts to studying the dynamics and trajectories in the two- or three-dimensional physical space that the fluid occupies. Namely, one studies the dynamics of the ordinary differential equations in the physical space:

$$
\left\{\begin{array}{l}
\frac{d x}{d t}=v(x, t), \\
\left.x\right|_{t=0}=x_{0} .
\end{array}\right.
$$


Here $v$ is the velocity field of the fluid, satisfying the Navier-Stokes equations or the quasigeostrophic equations in the context of this paper, $x$ is the physical location of the fluid parcel, and $t$ is the time.

The point of view of our theory is to classify the topological structure and its transitions of the instantaneous velocity field (i.e., streamlines in the Eulerian coordinates), treating the time variable as a parameter. Following the above viewpoint, Ma and Wang [16-18] developed a systematic geometrical theory of the velocity fields of two-dimensional incompressible flows, which is summarized as follows. One justification of this viewpoint is that (see below) given initial data, for a residual set (i.e., the intersection of a countable number of open and dense sets) of forcing, the instantaneous velocity field is structurally stable in an open and dense set of the time interval. Hence, the structural bifurcation can occur only on a nowhere dense set of time, and is a well-defined concept.

\section{A.2. Classification of Singularities of Divergence-Free Vector Fields}

Let $M \subset \mathbb{R}^{2}$ (or $M \subset S^{2}$ ) be a two-dimensional manifold with boundary, and $x=0 \in M$. For simplicity, we set

$$
D^{r}(T M)=\left\{v \in C^{r}(T M) \mid \operatorname{div} v=0\right\}
$$

for $r \geq 1$. Here $T M$ is the tangent bundle of $M$, and in the case where $M \subset \mathbb{R}^{2}, C^{r}(T M)=$ $\left(C^{r}(M)\right)^{2}$ containing all $C^{r}$ vector fields on $M$.

We consider a divergence-free vector field $v \in D^{r}(T M)(r \geq 1)$, and $x=0$ a nondegenerate singular point of $v$; i.e., the Jacobian $J(v(0))$ of $v$ at $x=0$ is nonsingular. In a neighborhood of $x=0, v$ can be expressed by

$$
v(x)=A x+g(x),
$$

where

$$
A=\left(\begin{array}{ll}
a_{11} & a_{12} \\
a_{21} & a_{22}
\end{array}\right), \quad g(x)=o(\|x\|) .
$$

Since $v$ is divergence-free, it is proved (see Proposition 2.1 in [16]) that an interior nondegenerate singular point must be either a center or a saddle point, and a nondegenerate singular point on the boundary must be a saddle point. The indices and physical meanings of these singular points are as follows:

1. When the eigenvalues of the matrix $A$ are purely imaginary numbers, the interior singularity $x=0$ is a center of the vector field (A.3) with index 1:

$$
\operatorname{ind}(v, 0)=1 .
$$

In fluid mechanics, $x=0$ corresponds to the center of a local circulation.

2. When the eigenvalues $\lambda_{1}$ and $\lambda_{2}$ of $A$ are real numbers, we have $\lambda_{1}=-\lambda_{2}>0$. In this case, $x=0$ is a saddle point with index -1 :

$$
\operatorname{ind}(v, 0)=-1
$$



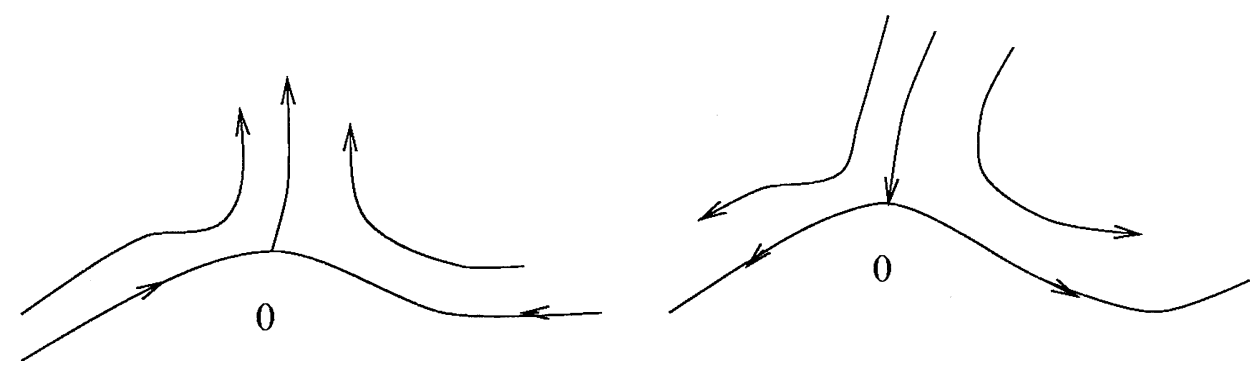

FIG. A.1. Saddle points on the boundary.

3. When $x=0 \in \partial M$, it has to be a saddle point of the vector field $v$ as shown in Fig. A.1. In this case, using the reflection principle we can extend the vector field across the boundary near $x=0$, and then define its index to be half of the extended field. Namely, the index is $-1 / 2$ :

$$
\operatorname{ind}(v, 0)=-\frac{1}{2}
$$

In the large-scale oceanic motion (resp. a general fluid motion), saddle points on the boundary are normally caused by a jet flowing toward or away from the coastal line or the boundary; see Fig. A.2.

\section{A.3. An Index Formula for Divergence-Free Vector Fields}

Let $M \subset \mathbb{R}^{2}$ (or $M \subset S^{2}$ ) be a compacted manifold with boundary with $k$ holes (see Fig. A.3). For the oceanic motion, the $k$ genus represents $k$ islands, and for the atmospheric motion, they represent the horizontal sections of $k$ mountains.

Let $v \in D^{r}(T M)(r \geq 1)$ be regular; i.e., all singular points are nondegenerate. Set

$C=$ the number of centers of $v$,

$S=$ the number of interior saddle points of $v$,

$B=$ the number of boundary saddle points of $v$.

Then we have the following index formula [17]

$$
C-S-\frac{1}{2} B=1-k \text {. }
$$

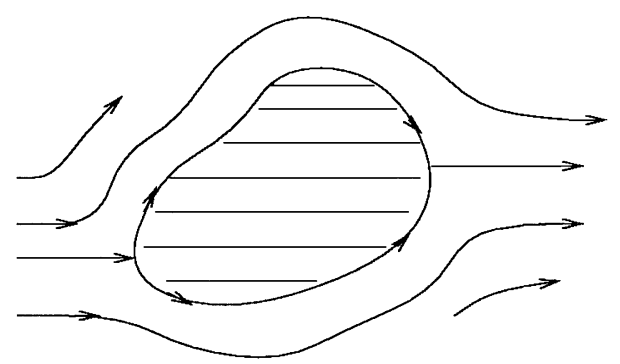

FIG. A.2. Flow passing through an island. 


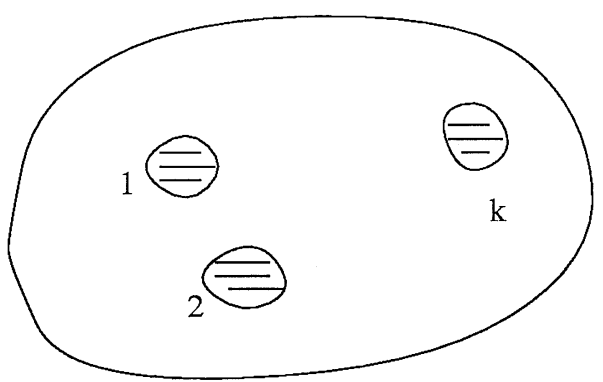

FIG. A.3. Domain $M$ with $k$ islands.

\section{A.4. Global Structural Classification of Divergence-Free Vector Fields}

DEFINITION A.1.

1. Let $p \in M$ be a center of $v$. The largest neighborhood $C$ of $p$ containing closed orbits is called a circle cell of the center $p$.

2. Given a closed orbit $\gamma$, let $B$ be the largest neighborhood of $\gamma$ containing only closed orbits. If any connected component of $\partial B$ is not a single point, then $B$ is called a circle band.

3. An orbit with its end points is called a saddle connection if its two end points are saddle points.

Then we have

THEOREM A.1 (Global Structural Classification [16]). Let $v \in D^{r}(T M)$ be a regular vector field. Then the topological set of orbits of $v$ consists of a finite number of connected components of circle cells, circle bands, and saddle connections.

\section{A.5. Topological Classification}

Two vector fields $v_{1}, v_{2} \in D^{r}(T M)$ are called topologically equivalent if there exists a homeomorphism $\phi: M \rightarrow M$, which maps orbits of $v_{1}$ to orbits of $v_{2}$ preserving the orientation.

THEOREM A.2 [16]. Let $v_{1}, v_{2} \in D^{r}(T M)$ be two regular divergence-free vector fields, and $\Gamma_{1}, \Gamma_{2}$ are the collections of saddle connections of $v_{1}$ and $v_{2}$, respectively. Then $v_{1}$ and $v_{2}$ are topologically equivalent if and only if $\Gamma_{1}$ and $\Gamma_{2}$ have the same topological structure.

This theorem tells us that the global topological structure of the flow orbits of a regular divergence-free vector field $v$ is completely determined by its saddle connection set.

\section{A.6. Global Structural Stability}

A divergence-free vector field $v$ is called incompressibly structurally stable if there exists a neighborhood $\mathcal{O} \subset D^{r}(T M)$ of $v$ such that any $u \in \mathcal{O}$ is topologically equivalent to $v$. Then we have

THEOREM A.3 (Global Structural Stability [16, 18]). A divergence-free vector field $v \in$ $D^{r}(T M)(r \geq 1)$ is (globally) incompressibly structurally stable (or for simplicity, structurally stable) if and only if 


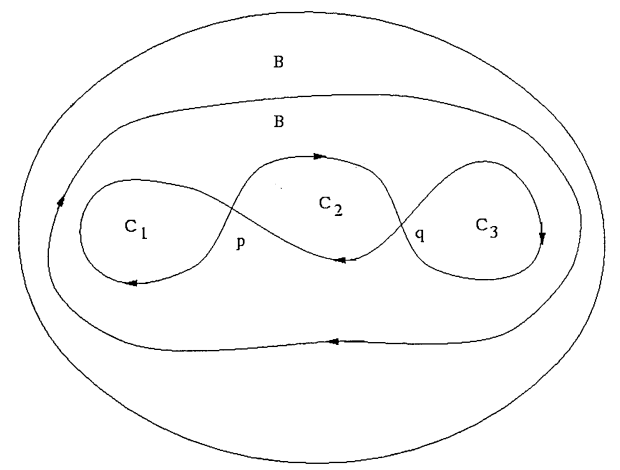

FIG. A.4. $C_{1}, C_{2}$, and $C_{3}$ are circle cells, $B$ is a circle band, and the orbits connected to $p$ or $q$ are saddle connections.

1. $v$ is regular,

2. all interior saddle points of $v$ are self-connected, and

3. each boundary saddle point is connected to boundary saddles on the same connected component of the boundary.

Moreover, the set of all structurally stable vector fields is open and dense in $D^{r}(T M)(r \geq 1)$.

Here we make a comparison with the classical structural stability theorem by Peixoto [24] for general vector fields (not necessarily divergence free). The conditions for structural stability and genericity in Peixoto's theorem are: (i) the field can have only a finite number of singularities and closed orbits (critical elements) which must be hyperbolic; (ii) there are no saddle connections; and (iii) the nonwandering set consists of singular points and closed orbits.

In Theorem A.3, the first condition here requires only regularity of the field and so it does not exclude centers which are not hyperbolic and excluded by (i) above. The second condition is of a completely different nature from the corresponding one in the Peixoto theorem. Namely, condition (ii) above excludes the possibility of saddle connections. In contrast, (2) amounts to saying that all interior saddles are self-connected! Namely, the interior saddles occur in graphs whose topological form is that of the number 8, the singularities themselves being hyperbolic. Condition (3) deals with singularities on the boundary. By this theorem it is easy to see that the flow pattern given in Fig. A.4 is NOT structurally stable since two saddle points $p$ and $q$ are connected, while both the flow patterns illustrated in Fig. 4.11 are structurally stable.

\section{A.7. Genericity of Structurally Stable Steady States of the 2D Navier-Stokes Equations}

Consider the following Navier-Stokes equations on a two-dimensional closed bounded domain $M$ :

$$
\begin{aligned}
& -\mu \Delta u+(u \cdot \nabla) u+\nabla p=f, \quad \text { in } \stackrel{M}{ } \subset \mathbb{R}^{2}, \\
& \operatorname{div} u=0, \quad \text { in } \stackrel{\circ}{M}, \\
& u_{n}=0,\left.\quad \frac{\partial u_{\tau}}{\partial n}\right|_{\partial M}=0, \quad \text { on } \partial M \text {. }
\end{aligned}
$$

Here $\stackrel{\circ}{M}$ stands for the interior of $M$. 
Set

$$
B^{r}(T M)=\left\{v \in D^{r}(T M)\left|\frac{\partial v_{\tau}}{\partial n}\right|_{\partial M}=0\right\},
$$

where $v_{n}=v \cdot n, v_{\tau}=v \cdot \tau$, and $n$ and $\tau$ are the unit normal and tangent vectors on $\partial M$, respectively. If $r=k+\alpha$ with $k \geq 0$ an integer and $0<\alpha<1$, then $v \in C^{r}(T M)$ means that $v \in C^{k}(T M)$ and all derivatives of $v$ up to order $k$ are $\alpha$-Hölder continuous. By definition, vector fields in $B^{r}(T M)$ satisfy the following free boundary conditions:

$$
\left.v_{n}\right|_{\partial M}=0,\left.\quad \frac{\partial v_{\tau}}{\partial n}\right|_{\partial M}=0 .
$$

Then we have (see [19]).

THEOREM A.4. For any $\mu>0$, there is an open and dense set $\mathcal{F} \subset C^{\alpha}(T M)(0<\alpha<1)$ such that for each $f \in \mathcal{F}$, the steady-state solutions $u \in X=B^{2+\alpha}(T M)$ of (A.5)-(A.7) are structurally stable in $X$.

Moreover, we can prove that for any given initial data and a residual set (i.e., the intersection of a countable number of open and dense sets) of forcing, the instantaneous velocity field is structurally stable in an open and dense set of the time interval. Therefore, generically, the structural bifurcation occurs only at a nowhere dense set of the time interval; therefore this fact justifies the structural bifurcation concept.

\section{A.8. A Remark on Applications}

We remark that the interior centers correspond to circulations, which are observable; boundary saddles correspond to jets moving toward or away from the boundary, which are also observable. By the index formula, the number of interior saddles can be easily determined. The structural classification theorem and the global structural theorem ensure that the global structure of the flow pattern is then uniquely determined by saddle connections. Moreover, given a divergence-free vector field, it is easy to verify the structural stability conditions in the structural stability theorem, which can be directly used in verifying the structural stability of the flow pattern.

\section{REFERENCES}

1. P. Berloff and S. Meacham, The dynamics of an equivalent-barotropic model of the wind driven circulation, J. Mar. Res. 55, 523 (1997).

2. P. Berloff and S. Meacham, The dynamics of a simple baroclinic model of the wind-driven circulation, J. Phys. Oceanogr. 28, 361 (1998).

3. K. Bryan, A numerical investigation of a nonlinear model of a wind-driven ocean, J. Atoms. Sci. 20, 594 (1963).

4. C. Canuto, M. Y. Hussaini, A. Quarteroni, and T. A. Zang, Spectral Methods in Fluid Dynamics (SpringerVerlag, New York/Berlin, 1987).

5. J. G. Charney, The dynamics of long waves in a baroclinic westerly current, J. Meteorol. 4, 135 (1947).

6. E. P. Chassignet, Vorticity dissipation by western boundary currents in the presence of outcropping layers, J. Phys. Oceanogr. 25, 242 (1995).

7. E. P. Chassignet and R. Bleck, The influence of the layer outcropping on the separation of boundary currents. Part i. The wind-driven experiments, J. Phys. Oceanogr. 23, 1485 (1993). 
8. A. J. Chorin, Numerical solution of the Navier-Stokes equations, Math. Comput. 22, 745 (1968).

9. D. B. Haidvogel, J. C. McWilliams, and P. R. Gent, Boundary current separation in a quasi-geostrophic, eddy-resolving ocean circulation model, J. Phys. Oceanogr. 11, 557 (1992).

10. W. R. Holland, The role of mesoscale eddies in the general circulation of the ocean, J. Phys. Oceanogr. 8, 363 (1978).

11. W. R. Holland and P. B. Rhines, An example of eddy-induced ocean circulation, J. Phys. Oceanogr. 10, 1010 (1980).

12. S. Jiang, F.-F. Jin, and M. Ghil, Multiple equilibria, periodic, and aperiodic solutions in a wind-driven, doublegyre, shallow-water model, J. Phys. Oceanogr. 25, 764 (1995).

13. J. Van Kan, A second-order accurate pressure-correction scheme for viscous incompressible flow, SIAM J. Sci. Stat. Comput. 7, 870 (1986).

14. J. L. Lions, R. Temam, and S. Wang, On mathematical problems for the primitive equations of the ocean: The mesoscale midlatitude case, Nonlinear Anal., to appear.

15. R. E. Lynch, J. R. Rice, and D. H. Thomas, Direct solution of partial differential equations by tensor product methods, Numer. Math. 6(1), 185 (1964).

16. T. Ma and S. Wang, Structural classification and stability of divergence-free vector fields, submitted.

17. T. Ma and S. Wang, Generalized Poincaré-Hopf index formula and its applications to 2-D incompressible flows, submitted.

18. T. Ma and S. Wang, Dynamics of two-dimensional incompressible flows, Appl. Math. Lett. 12, 39 (1999).

19. T. Ma and S. Wang, The geometry of the stream lines of steady states of the Navier-Stokes equations, in Contemporary Mathematics (Am. Math. Soc., Providence, 1999), p. 238.

20. S. Meacham and P. Berloff, Instabilities of a steady, barotropic, wind-driven circulation, J. Mar. Res. 55, 407 (1997).

21. T. M. Ozgokmen and E. P. Chassignet, On the emergence of inertial gyres in a two-layer quasi-geostrophic ocean model, J. Phys. Oceanogr. 28, 461 (1997).

22. J. Pedlosky, Geophysical Fluid Dynamics, 2nd ed. (Springer-Verlag, New-York, 1987).

23. J. Pedlosky, Ocean Circulation Theorey (Springer-Verlag, New-York, 1996).

24. M. Peixoto, Structural stability on two dimensional manifolds, Topology 1, 101 (1962).

25. Jie Shen, Efficient spectral-Galerkin method. I. Direct solvers for second- and fourth-order equations by using Legendre polynomials, SIAM J. Sci. Comput. 15, 1489 (1994).

26. Jie Shen, Efficient spectral-Galerkin method. II. Direct solvers for second- and fourth-order equations by using Chebyshev polynomials, SIAM J. Sci. Comput. 16, 74 (1995).

27. Jie Shen, Efficient Chebyshev-Legendre Galerkin methods for elliptic problems, in Proceedings, ICOSAHOM'95, edited by A.V. Ilin and R. Scott, pp. 233-240. [Houston J. Math. (1996)]

28. Piotr K. Smolarkiewicz and Len G. Margolin, MPDATA: A finite-difference solver for geophysical flows, J. Comput. Phys. 140(2), 459 (1998).

29. S. Speich, H. Dijkstra, and M. Ghil, Successive bifurcations in a shallow-water model, applied to the winddriven ocean circulation, Nonlin. Proc. Geophys. 2, 241 (1995).

30. S. Speich and M. Ghill, Interannual variability of the mid-latitude oceans: A new source of climate variability? Sistema Terra 3(3), 33 (1994).

31. H. Stommel, The westward intensification of the wind-driven ocean currents, Trans. Am. Geosphys. Union 29, 202 (1948).

32. R. Temam, Sur l'approximation de la solution des équations de Navier-Stokes par la méthode des pas fractionnaires ii, Arch. Rat. Mech. Anal. 33, 377 (1969).

33. G. Veronis, Wind-driven ocean circulation, part i and part ii, Deep-Sea Res. 13, 31 (1966). 